Accelerator Division

Alternating Gradient Synchrotron Department

BROOKHAVEN NATIONAL LABORATORY

Upton, New York 11973

Accelerator Division

Technical Note

AGS/AD/Tech. Note. No. 422

TRANSVERSE BUNCHED BEAM INSTABILITY

S. Y. Zhang

November 14, 1995 


\title{
TRANSVERSE BUNCHED BEAM INSTABILITY
}

\author{
S. Y. Zhang
}

\begin{abstract}
A transverse bunched beam instability formalism is presented, which is used to analyse the transverse Robinson type, the head-tail, and the coupled bunch instabilities. The formalism can also be used for the transverse mode coupling study, and its simplified version can include the Landau damping, and therefore to estimate the instability threshold. The presentation is focused on the beam signal spectrum, the impedance, and their samplings.
\end{abstract}




\section{TRANSVERSE BUNCHED BEAM INSTABILITY}

\section{Introduction}

In this article, a transverse bunched beam instability formalism is presented. Based on the particle distribution, a set of orthogonal polynomials is used for the expansion of radial modes, which enables the formalism in dealing with the transverse Robinson instability, the head tail instability, the coupled bunch instability, and the transverse mode coupling as well.

From the formalism it is shown that the envelope product of the beam signal spectrum and the impedance is an important factor in determining the beam instability. Also, since the beam signal is periodic, the real beam signal spectrum is a train of pulses, which implies that the effective impedance is determined by this samplings. In this article, the mechanism of the instability is studied by focusing on these two aspects.

After presenting the formalism and the solutions, the beam signal spectrum and the impedance will be studied. The result, together with the signal samplings, is used for the single bunch instability study. Finally the multiple bunch instability and some application to the AGS are presented.

In a simplified version, the first orthogonal polynomial can be used, which reduces the dynamic equation into a scale equation, then the Landau damping can be included and the growth rate and instability threshold can be estimated. Most issues related to the simplified version and the transverse mode coupling will be presented in separate works.

\section{Sacherer Integral Equation for Transverse Motion}

Consider the four dimensional phase space of longitudinal and transverse motion. The Vlasov equation is [1],

$$
\frac{\partial \psi}{\partial t}+\dot{x} \frac{\partial \psi}{\partial x}+\ddot{x} \frac{\partial \psi}{\partial \dot{x}}+\dot{\phi} \frac{\partial \psi}{\partial \phi}+\ddot{\phi} \frac{\partial \psi}{\partial \dot{\phi}}=0
$$

where $\psi(x, \dot{x}, \phi, \dot{\phi}, t)$ is the normalized phase space density, $\phi$ is the phase deviation of the particle and $x$ is the transverse displacement. Neglecting longitudinal perturbation, the synchrotron oscillation is described as,

$$
\ddot{\phi}+\omega_{S}^{2} \phi=0
$$

where $\omega_{S}$ is the synchrotron oscillation frequency. The betatron oscillation is described as,

$$
\ddot{x}+\omega_{\beta}^{2} x=\frac{F_{x}}{m_{0} \gamma}
$$


where $\omega_{\beta}$ is the betatron oscillation frequency. $F_{x}$ is the wake force due to the field induced by the perturbation, and $m_{0} \gamma$ is the relativistic mass of the particle.

We write the phase space co-ordinates $\left(\phi, \dot{\phi} / \omega_{S}\right)$ in the polar phase space $(r, \theta)$,

$$
\begin{gathered}
\phi=r \cos \theta \\
\dot{\phi} / \omega_{S}=r \sin \theta
\end{gathered}
$$

and similarly for the transverse phase space,

$$
\begin{gathered}
\phi_{x}=r_{x} \cos \theta_{x} \\
\dot{\phi}_{x} / \omega_{\beta}=r_{x} \sin \theta_{x}
\end{gathered}
$$

Accordingly, the Vlasov equation (2-1) is written as,

$$
\frac{\partial \psi}{\partial t}-\omega_{S} \frac{\partial \psi}{\partial \theta}-\omega_{\beta} \frac{\partial \psi}{\partial \theta_{x}}=-\frac{F_{x}}{m_{0} \gamma} \frac{\partial \psi}{\partial \dot{x}}
$$

Let the particle distribution be,

$$
\psi=\psi_{0}(r) \psi_{x 0}\left(r_{x}\right)+\psi_{p}(r, \theta) \psi_{x p}\left(r_{x}, \theta_{x}\right) e^{j \omega t}
$$

where we assume that the unperturbed stationary distribution depends only on radial and the perturbed terms in the two directions are not cross-affected. Using

$$
\frac{\partial \psi_{x 0}}{\partial \dot{x}} \approx \frac{\sin \theta_{x}}{\omega_{\beta}} \frac{d \psi_{x 0}}{d r_{x}} \psi_{0}
$$

the linearized Vlasov equation becomes,

$$
\left(j \omega \psi_{p} \psi_{x p}-\omega_{S} \psi_{x p} \frac{\partial \psi_{p}}{\partial \theta}-\omega_{\beta} \psi_{p} \frac{\partial \psi_{x p}}{\partial \theta_{x}}\right) e^{j \omega t}=-\frac{F_{x}}{m_{0} \gamma} \frac{\sin \theta_{x}}{\omega_{\beta}} \frac{d \psi_{x 0}}{d r_{x}} \psi_{0}
$$

Assuming a rigid transverse dipole oscillation in phase space with radius $D$,

$$
\psi_{x p}\left(r_{x}, \theta_{x}\right)=\psi_{x 0}\left(r_{x}-D e^{j \theta_{x}}\right)-\psi_{x 0}\left(r_{x}\right) \approx-D e^{j \theta_{x}} \frac{d \psi_{x 0}}{d r_{x}}
$$

and letting

$$
\sin \theta_{x} \approx \frac{e^{j \theta_{x}}}{2 j}
$$

then the equation (2-11) becomes,

$$
\left(j \omega \psi_{p}-\omega_{S} \frac{\partial \psi_{p}}{\partial \theta}-j \omega_{\beta} \psi_{p}\right) D e^{j \omega t}=\frac{F_{x}}{2 j m_{0} \gamma} \frac{\psi_{0}}{\omega_{\beta}}
$$

Consider the longitudinal perturbation distribution,

$$
\psi_{p}(r, \theta)=\sum_{m^{\prime}=-\infty}^{\infty} R^{\left(m^{\prime}\right)}(r) e^{j m^{\prime} \theta}
$$

where $R^{\left(m^{\prime}\right)}(r)$ is the radial function with the $m^{\prime}$ th azimuthal mode. The corresponding line density is defined as, 


$$
\lambda(\phi)=\int_{-\infty}^{\infty} \psi_{p}\left(\phi, \dot{\phi} / \omega_{S}\right) d \dot{\phi} / \omega_{S}
$$

which can be Fourier expanded as,

$$
\lambda(\phi)=\frac{1}{2 \pi} \sum_{p=-\infty}^{\infty} \Lambda(p) e^{j p \phi}
$$

where the spectrum $\Lambda(p)$ is defined as,

$$
\Lambda(p)=\int_{-\infty}^{\infty} \lambda(\phi) e^{-j p \phi} d \phi
$$

If we define the Hankel spectrum of the radial function $R^{(m)}(r)$ as,

$$
\Lambda^{(m)}(p)=\int_{-\infty}^{\infty} R^{(m)}(r) J_{m}(p r) r d r
$$

then the relation between the Fourier spectrum of the line density, expanded on the phase deviation $\phi$, and the Hankel spectrum of the radial function is shown as,

$$
\Lambda(p)=2 \pi \sum_{m^{\prime}=-\infty}^{\infty} j^{-m^{\prime}} \Lambda^{\left(m^{\prime}\right)}(p)
$$

Using the relation

$$
F=e E
$$

the wake force $F_{x}$ is written as,

$$
F_{x}=-\frac{j e D I_{0}}{2 \pi R} e^{j \omega t} \sum_{p=-\infty}^{\infty} Z_{T}(p) \Lambda(p) e^{j p \phi}
$$

where $Z_{T}(p)$ is the transverse impedance sampled at the frequency $p \omega_{0}, \omega_{0}$ is the revolution frequency, $R$ is the machine radius, and the average beam current $I_{0}$ is proportional to the number of particles $N$,

$$
I_{0}=\frac{N e \omega_{0}}{2 \pi}
$$

Substituting (2-15) and (2-22) into (2-14), multiplying $e^{-j m \theta}$, and integrating over $\theta$, we have,

$$
j\left(\omega-\omega_{\beta}-m \omega_{S}\right) R^{(m)}(r)=-\frac{e I_{0} \psi_{0}}{4 \pi R m_{0} \gamma \omega_{\beta}} \sum_{p=-\infty}^{\infty} Z_{T}(p) \Lambda(p) j^{m} J_{m}(p r)
$$

where the relations

$$
\int_{0}^{2 \pi} e^{j(n-m) \theta} d \theta=2 \pi \delta_{n, m}
$$

and 


$$
\int_{0}^{2 \pi} e^{-j(m \theta-p r \cos \theta)} d \theta=2 \pi j^{m} J_{m}(p r)
$$

have been used.

\section{Solution of the Integral Equation}

To solve the transverse dynamic equation (2-24), we define a set of orthogonal polynomials according to a weight function $W(r)$ for the azimuthal mode $m[2,3]$,

$$
\int_{0}^{\infty} W(r) f_{k}^{(m)}(r) f_{\ell}^{(m)}(r) r d r=\delta_{k, \ell}
$$

Using the orthogonal polynomials, the radial function can be written as,

$$
R^{(m)}(r)=W(r) \sum_{k^{\prime}=0}^{\infty} \alpha_{k^{\prime}}^{(m)} f_{k^{\prime}}^{(m)}(r)
$$

Define the Hankel spectrum for the orthogonal polynomial,

$$
\Lambda_{k}^{(m)}(p)=\int_{0}^{\infty} W(r) f_{k}^{(m)}(r) J_{m}(p r) r d r
$$

then we have,

$$
\Lambda^{(m)}(p)=\sum_{k^{\prime}=0}^{\infty} \alpha_{k^{\prime}}^{(m)} \Lambda_{k^{\prime}}^{(m)}(p)
$$

The Bessel function $J_{m}(p r)$ can be expanded by the orthogonal polynomials and the associated Hankel spectra,

$$
J_{m}(p r)=\sum_{k^{\prime}=0}^{\infty} \Lambda_{k^{\prime}}^{(m)}(p) f_{k^{\prime}}^{(m)}(r)
$$

Let

$$
W(r)=\psi_{0}(r)
$$

Substituting (3-2), (3-4) and (3-5) into (2-24), we obtain,

$$
\begin{gathered}
\left(\omega-\omega_{\beta}-m \omega_{S}\right) W(r) \sum_{k^{\prime}=0}^{\infty} \alpha_{k^{\prime}}^{(m)} f_{k^{\prime}}^{(m)}(r)=\frac{j^{m+1} e I_{0}}{2 R m_{0} \gamma \omega_{\beta}} W(r) \sum_{p=-\infty}^{\infty} Z_{T}(p) \\
\times \sum_{k^{\prime}, \ell^{\prime}=0}^{\infty} \Lambda_{k^{\prime}}^{(m)}(p) f_{k^{\prime}}^{(m)}(r) \sum_{m^{\prime}=-\infty}^{\infty} j^{-m^{\prime}} \alpha_{\ell^{\prime}}^{\left(m^{\prime}\right)} \Lambda_{\ell^{\prime}}^{\left(m^{\prime}\right)}(p)
\end{gathered}
$$

Multiplying (3-7) by $f_{k}^{(m)}(r) r$ for $k=0, \ldots, \bar{k}$, integrating over $r$, and using (3-1), we finally have the following equation,

$$
\left(\omega-\omega_{\beta}-m \omega_{S}\right) \alpha^{(m)}=\frac{j^{m+1} e I_{0}}{2 R m_{0} \gamma \omega_{\beta}} \sum_{p=-\infty}^{\infty} Z_{T}(p) \sum_{k^{\prime}, \ell^{\prime}=0}^{\infty} \Lambda_{k^{\prime}}^{(m)}(p) \sum_{m^{\prime}=-\infty}^{\infty} j^{-m^{\prime}} \alpha_{\ell^{\prime}}^{\left(m^{\prime}\right)} \Lambda_{\ell^{\prime}}^{\left(m^{\prime}\right)}(p)
$$


where the vector $\alpha^{(m)}$ is defined as,

$$
\alpha^{(m)}=\left[\begin{array}{c}
\alpha_{0}^{(m)} \\
\vdots \\
\alpha_{\bar{k}}^{(m)}
\end{array}\right]
$$

where $k$ is truncated at $\bar{k}$. Define the system matrix,

$$
\mathbf{M}^{\left(m, m^{\prime}\right)}=\frac{j^{m+1-m^{\prime}} e I_{0}}{2 R m_{0} \gamma \omega_{\beta}}\left[\begin{array}{ccc}
\sum_{p=-\infty}^{\infty} Z_{T}(p) \Lambda_{0}^{(m)}(p) \Lambda_{0}^{\left(m^{\prime}\right)}(p) & \cdots & \sum_{p=-\infty}^{\infty} Z_{T}(p) \Lambda_{0}^{(m)}(p) \Lambda_{\bar{k}}^{\left(m^{\prime}\right)}(p) \\
\vdots & \vdots & \vdots \\
\sum_{p=-\infty}^{\infty} Z_{T}(p) \Lambda_{\bar{k}}^{(m)}(p) \Lambda_{0}^{\left(m^{\prime}\right)}(p) & \cdots & \sum_{p=-\infty}^{\infty} Z_{T}(p) \Lambda_{\bar{k}}^{(m)}(p) \Lambda_{\bar{k}}^{\left(m^{\prime}\right)}(p)
\end{array}\right]
$$

Taking only the azimuthal modes $m$ and $m^{\prime}$, the equation (3-8) can be written as,

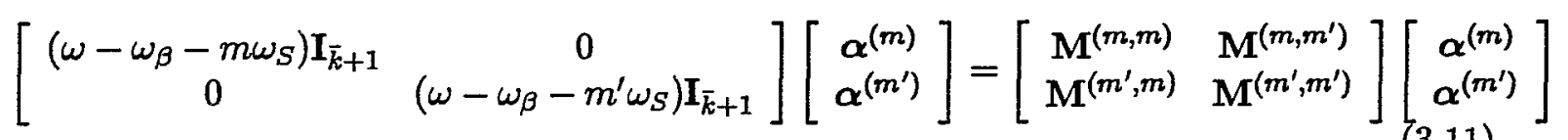

where $I$ is the identity matrix. The equation (3-11) can be solved as an eigenvalue problem.

Finally, the spectra of orthogonal polynomials $\Lambda_{k}^{(m)}(p)$ for a Gaussian distribution with a half bunch length $\phi=\pi / 2$ are shown in Fig.1, where the azimuthal modes $m$ is from 0 to 2 , and the number of orthogonal polynomials is 3 .

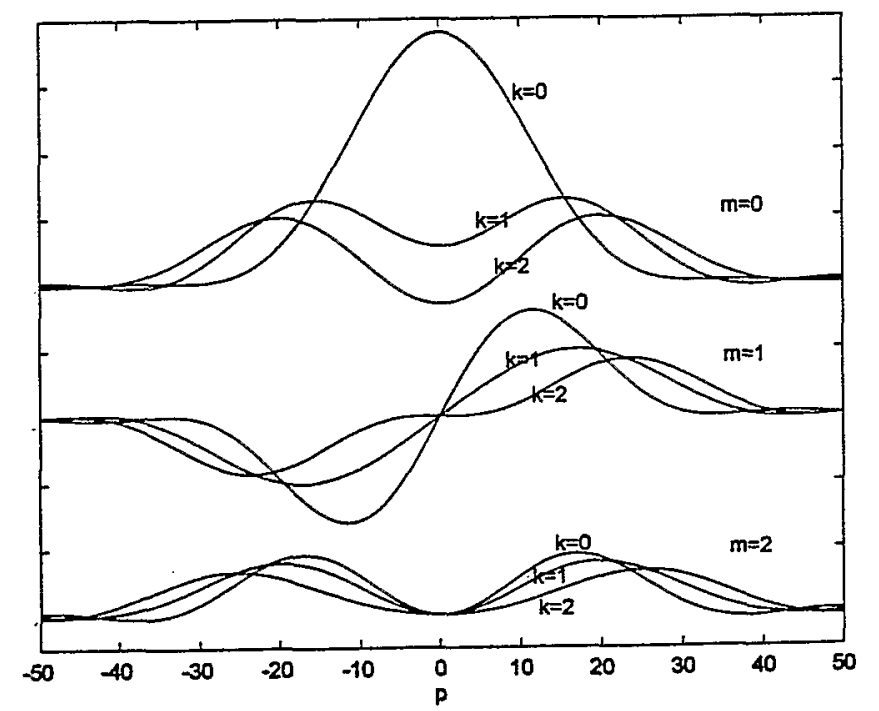

Fig.1. Spectra of Orthogonal Polynomials of Gassian

The power spectra $\Lambda_{0}^{(m)}(p) \Lambda_{0}^{(m)}(p)$ of the Gaussian distribution with half bunch length $\phi=\pi / 2$ and $\phi=\pi / 4$ of the first orthogonal polynomials are shown in Fig.2 for comparison. Note that the half bandwidth of the power spectrum equals to $\pi h / \phi$. In Figs.1 and 2 , the AGS harmonic number $h=8$ is used, one may find in Fig.2 that the half bandwidths are 16p and $32 p$, respectively. If we take the revolution frequency as $350 \mathrm{KHz}$, then the spectrum 
half bandwidths corresponding to the half bunch lengths $\phi=\pi / 2$ and $\phi=\pi / 4$ are $5.6 \mathrm{MHz}$ and $11.2 \mathrm{MHz}$, respectively.

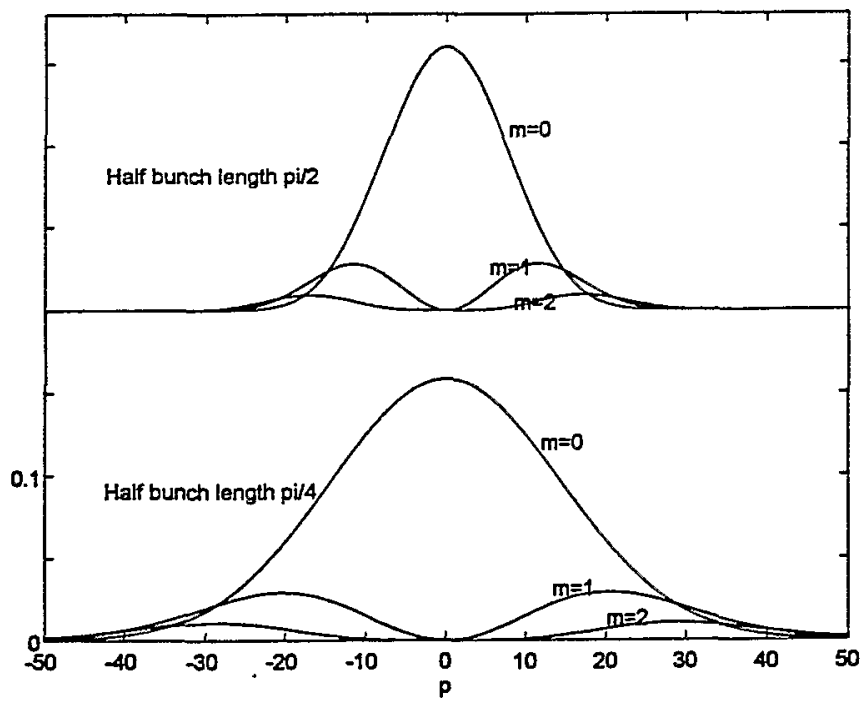

Fig.2. Power Spectra of First Orthogonal Polynomials

\section{Transverse Impedance}

In this section, we will present the transverse impedances of the resistive wall, the space charge effect, and the broad band and narrow band resonators.

\subsection{Resistive wall impedance}

The transverse resistive wall impedance is defined as,

$$
Z_{T}(\omega)=(\operatorname{sgn}(\omega)+j) \frac{R Z_{0} \delta_{s}}{b^{3}}
$$

where $R$ is the machine radius, $Z_{0}$ is the impedance in free space, $377 \Omega, b$ is the radius of the vacuum chamber, and $\delta_{s}$ is the skin depth at the frequency $\omega$,

$$
\delta_{s}=\sqrt{\frac{2 \rho}{\mu_{0}|\omega|}}
$$

where $\rho$ is the resistivity of the vacuum chamber, for steel it can be $\rho=10^{-6} \Omega m$, and $\mu_{0}=4 \pi \times 10^{-7} \mathrm{H} / \mathrm{m}$ is the permeability of free space.

For the AGS, $R=128 \mathrm{~m}$, and the vacuum chamber is $15.2 \mathrm{~cm} \times 7 \mathrm{~cm}$, therefore the vertical radius is taken as $b=3.5 \mathrm{~cm}$. At the injection, the revolution frequency is $344 \mathrm{KHz}$. The transverse resistive wall impedance at the revolution frequency is $Z_{T}\left(\omega_{0}\right)=0.97(1+j) M \Omega / m$. Since that the impedance reduces in a rate of the square root of the frequency, therefore the most influential part of the resistive wall impedance is at the low frequencies.

Note that we considered only a smooth vacuum chamber and nothing else. Also we assumed that the skin depth is less than the thickness of the chamber, which is taken as $1.5 \mathrm{~mm}$. Should the skin depth be larger than the chamber thickness, the impedance would be reversely proportional to the frequency, rather than the square root of the frequency. 
Finally, the resistivity of the AGS vacuum chamber material can be $\rho=1.2 \times 10^{-6} \Omega m$ and a modification factor should be added to the vertical radius of the chamber, since the chamber is not a circular pipe. All of these pose some unknown factors to the total impedance. Therefore a measurement is needed for a better knowledge of this impedance.

\subsection{Space charge effect}

The space charge effect can be represented by an impedance defined as,

$$
Z_{T}(\omega)=-j \frac{R Z_{0}}{\beta^{2} \gamma^{2}}\left(\frac{1}{a^{2}}-\frac{1}{b^{2}}\right)
$$

where $a$ is the average radius of the beam. For AGS injection, we take $a=2 \mathrm{~cm}, \beta=0.93$, and $\gamma=2.7$, then the space charge impedance is $Z_{T}(\omega)=-j 12.9 M \Omega / m$, which is independent of the frequency, and can be seen as the negative inductance.

Note that the transverse space charge impedance is defined as proportional to the difference between the incoherent and coherent tune shift [4], and the incoherent tune shift, affected by the factor $1 / a^{2}$, is much larger than the coherent tune shift, affected by $1 / b^{2}$. It can be shown that in the transverse beam dynamic equation (2-24) the incoherent tune shift is cancelled from the both side of the equation, and only the coherent tune shift is effective to the beam coherent motion. This is different from the counterpart of the longitudinal beam motions. The space charge effect itself will not introduce instabilities, however, if the coherent tune shift is large, it can affect the Landau damping. In solving the beam dynamic equation, if the beam instability is concerned then only the impedance of the coherent space charge effect needs to be applied, not the one in (4-3).

\subsection{Broad band resonator}

In general a resonator type impedance can be written as,

$$
Z_{T}(\omega)=\frac{R_{T}}{1+j Q\left(\omega / \omega_{R}-\omega_{R} / \omega\right)}
$$

where $R_{T}$ is the transverse resistance, $Q$ is the quality factor and $\omega_{R}$ is the resonant frequency. The most important broad band resonator type impedance is the one caused by the vacuum chamber discontinuities, which is roughly modeled as a resonator with $\omega_{R} \approx c / b$, and $Q=1$ [5]. At the AGS, this resonant frequency is about $1.3 G H z$. At the low frequency, this impedance appears to be inductive, which gives rise to a coherent frequency shift, and therefore affects the Landau damping. The parameters of this impedance is an object of measurements.

\subsection{Narrow band resonator}

This type of impedance includes mainly the RF cavities. Usually the longitudinal impedance of the cavities is known, therefore a means to translate the longitudinal impedance into a transverse one is of interest. There is a general relation between the two impedances [6],

$$
Z_{T}(\omega)=\frac{2 c}{b^{2}} Z_{L}(\omega) / \omega
$$


where $Z_{L}(\omega)$ is the longitudinal impedance. This equation can be applied to broad band impedance, it fails, however, for narrow band impedance. It is claimed that the PanofskyWenzel theorem can be used for narrow band impedance [7],

$$
Z_{T}(\omega)=c Z_{L}(\omega) / \omega
$$

The application of this relation often generates unsatisfied results. Therefore, measurements are always required.

\section{Chromatic Effect}

One of the most important differences of the transverse motion from the longitudinal counterpart is the chromatic effect. The machine chromaticity $\xi$ is defined in the following relation,

$$
\frac{\omega_{\beta}-\omega_{\beta 0}}{\omega_{\beta 0}}=\xi \frac{\Delta p}{p_{0}}
$$

where $\omega_{\beta}$ and $\omega_{\beta 0}$ are the betatron and nominal betatron frequencies, respectively, and $\Delta p$ is the momentum deviation. Meanwhile, the frequency slip factor $\eta$ is defined in

$$
\frac{\omega-\omega_{0}}{\omega_{0}}=-\eta \frac{\Delta p}{p_{0}}
$$

where $\omega$ and $\omega_{0}$ are the revolution and nominal revolution frequencies, respectively.

These two relations give rise to an important result,

$$
\frac{\omega_{\beta}-\omega_{\beta 0}}{\omega_{\beta 0}}=-\frac{\xi}{\eta} \frac{\omega-\omega_{0}}{\omega_{0}}
$$

What can be learned from this equation is that the betatron frequency variation, affected by the chromaticity, is proportional to the revolution frequency variation. Since the revolution frequency variation is directly related to the synchrotron phase deviation $\phi$, one finds that the betatron frequency is no longer constant during the bunch passage. If we define the chromatic frequency

$$
\omega_{\xi}=\frac{\xi}{\eta} \omega_{\beta 0}
$$

then the beam line density is further modulated by $e^{j \omega_{\xi} \tau}$, where $\tau$ is the bunch delay time, this means that the beam signal envelope has a frequency shift with the chromatic frequency $\omega_{\xi}$. This problem can also be looked from the betatron phase modulation of the beam signal. Since the delay time $\tau$ is related to the phase deviation $\phi$ by,

$$
\tau=-\frac{\phi}{\omega_{0}}
$$

substituting this relation into $e^{j \omega_{\xi} \tau}$, the perturbation distribution defined in (2-15) becomes phase deviation dependent,

$$
\psi_{p}(r, \theta, \phi)=\sum_{m^{\prime}=-\infty}^{\infty} R^{\left(m^{\prime}\right)}(r) e^{j m^{\prime} \theta} e^{-j \frac{\omega_{\xi}}{\omega_{0}} \phi}
$$


Therefore the sampling number $p$ in the Hankel spectrum of the orthogonal polynomial in (3-3) will be replaced by

$$
p^{\prime}=p-\frac{\omega_{\xi}}{\omega_{0}}
$$

Note that in the equations (5-5)-(5-7), if the harmonic number $h \neq 1$ is considered, then $\omega_{0}$ should be replaced by $h \omega_{0}$. The more intuitive way to understand the implication of (5-7) is by simply considering that the power spectrum of the signal shifts by the chromatic frequency $\omega_{\xi}$. This affects the product of the signal spectrum and the impedance, and hence the instabilities.

\section{Single Bunch Instabilities}

\subsection{Beam dynamic equation}

We consider only the azimuthal mode $m$, and neglect the mode coupling. The following simplified equation of (3-11) can be used,

$$
\left(\omega-\omega_{\beta}-m \omega_{S}\right) \alpha^{(m)}=\mathbf{M}^{(m, m)} \alpha^{(m)}
$$

To further simplify the equation, we keep only the first orthogonal polynomial. If the bunch is not too short and the high frequency impedance is not significant, then the error generated by the simplification can be acceptable. Thus the equation (6-1) becomes,

$$
\omega-\omega_{\beta}-m \omega_{S}=\frac{j e I_{0}}{2 R m_{0} \gamma \omega_{\beta}} \sum_{p=-\infty}^{\infty} Z_{T}(p) \Lambda_{0}^{(m)}\left(p^{\prime}\right) \Lambda_{0}^{(m)}\left(p^{\prime}\right)
$$

From the previous study, the envelopes of the spectra of the signal and the impedance have been determined. Since the beam signal is always periodic with the revolution period $T=2 \pi / \omega_{0}$, for single bunch the real spectrum of the signal in fact is a train of pulses, whose amplitude is determined by the corresponding envelope. Therefore only the impedance at this train of pulses is effective, as shown in (6-2). The rules of the sampling are determined by the following factors.

1. For exact periodic signals with the revolution frequency $\omega_{0}, p$ represents samplings at $p \omega_{0}$.

2. If synchrotron oscillation is considered, then the signals are not exact periodic, but oscillating around the synchronous phase. Now $p$ represents samplings at $p w_{0}+m \omega_{S}$.

3. For the transverse dipole motion considered in Section $2, p$ represents samplings at $p \omega_{0}+\omega_{\beta}+m \omega_{S}$. Since only the samplings change, also the summation of $p$ is endless in both directions, the integer part of the betatron tune can be disregarded. It is noted that the fractional part of the betatron makes the situation different from the longitudinal case.

4. Finally, the nonzero chromaticity introduces a modulation of $e^{j \omega_{\xi} \tau}$ to the signal, which shifts the envelope of the signal. This fact is shown in (6-2) by the notation $p^{\prime}$ in the signal spectrum, which represents samplings at $p \omega_{0}-\omega_{\xi}+\omega_{\beta}+m \omega_{S}$, in a more general case of $h \neq 1$, it becomes $p \omega_{0}-\omega_{\xi} / h+\omega_{\beta}+m \omega_{S}$. 


\subsection{Transverse Robinson instability}

In the following, we use equation (6-2) to study several type of instabilities. Since the power spectra of the beam signal is always real, the imaginary part of the impedance generates the frequency shift and the real part of the impedance is relevant to damping or antidamping. Here the attention will be paid to the real part of the impedance.

Corresponding to the longitudinal Robinson instability, there is a transverse counterpart. The main difference between the two can be,

1. For longitudinal case, the mode $m=1$ is dominant, and for transverse the mode $m=0$ is dominant.

2. For longitudinal, the samplings are at $p \omega_{0}+m \omega_{S}$, and for transverse they are at $p \omega_{0}+\omega_{\beta}+m \omega_{S}$.

Consider first a narrow band resonator, such as an RF cavity and its higher order modes. The first difference shown above is not important in this case, but the second one affects the stability criterion. It is known that in the longitudinal case the samplings are shifted by $\omega_{S}$ from the revolution frequency, and the Robinson instability is determined by the RF cavity detuning direction. In the transverse case, the samplings are shifted by the fractional part of the betatron frequency, which is usually much larger than the synchrotron frequency. Thus, the transverse Robinson instability criterion is affected by whether the fractional betatron tune is larger or smaller than $1 / 2$, depending on the location of the resonant frequency. Also we may notice that for transverse, there is no difference above or below transition, as that for longitudinal. In Fig.3, we show an example, where a fractional tune $\nu_{f}=0.2$, and the samplings are indicated by long bars. This situation is stable, however, if we let $\nu_{f}=0.8$ it becomes unstable. Also we may observe that the change of the resonant frequency may affect the instabilities.

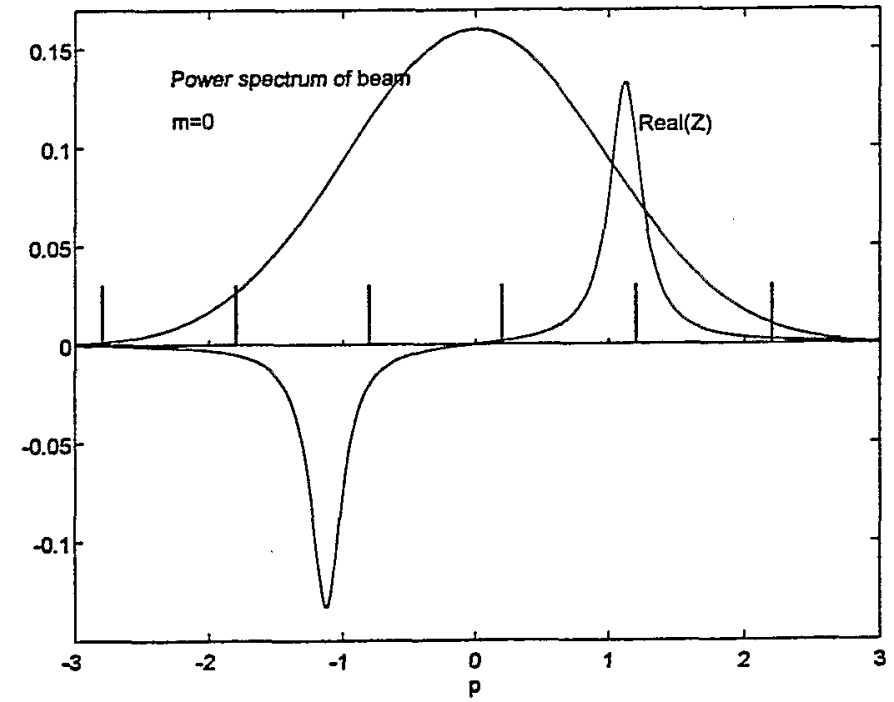

Fig.3. Transverse Robinson Instability, Narrow Band Resonator

The resistive wall impedance can be seen as an extension of the resonator with a very low resonant frequency. Since the signal spectrum of $m=1$ vanishes at the low frequency, this impedance is not effective for the longitudinal instability. It is however very important 
for the transverse counterpart where $m=0$ mode is dominant. For this impedance it is clear that if the fractional betatron tune is smaller than $1 / 2$, the system is stable, and otherwise unstable. In Fig.4, an example with $\nu_{f}=0.2$ is shown, the system is stable.

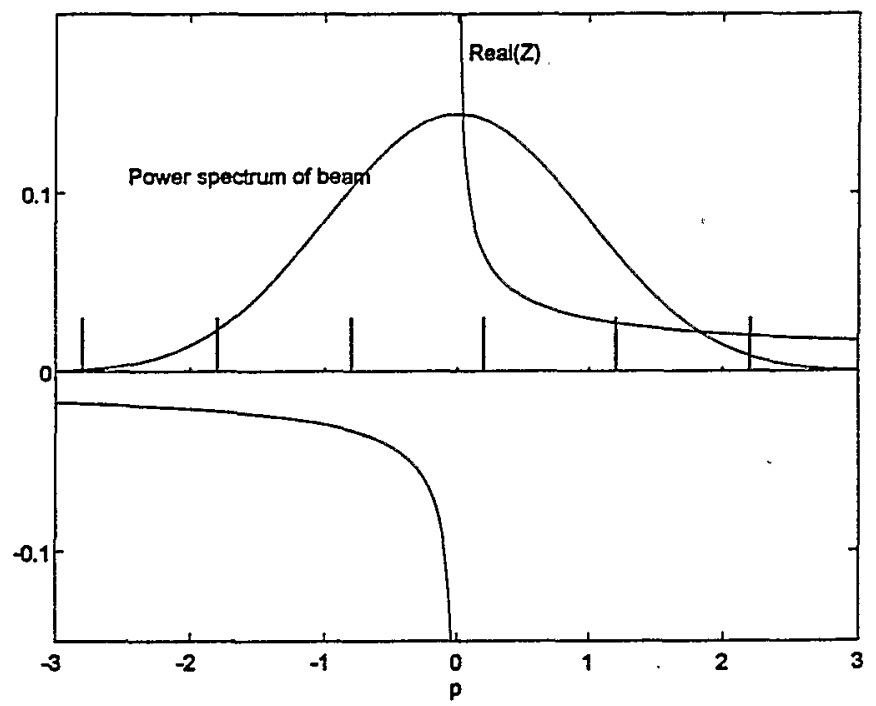

Fig.4. Transverse Robinson Instability, Resistive Wall

\subsection{Head tail instability}

With a non-zero chromaticity, the signal spectrum will shift as discussed previously. In general, if $\omega_{\xi}>0$, i.e. above transition $\xi>0$ and below transition $\xi<0$, then the system is stable, and vice versa. For higher order modes $m>0$, this may not be true. Also we note a possible conflict between the Robinson type instability and the head tail instability. The final result depends on the total effect including all these factors. In Fig.5, an example with $\omega_{\xi}>0$ is shown, the system is stable.

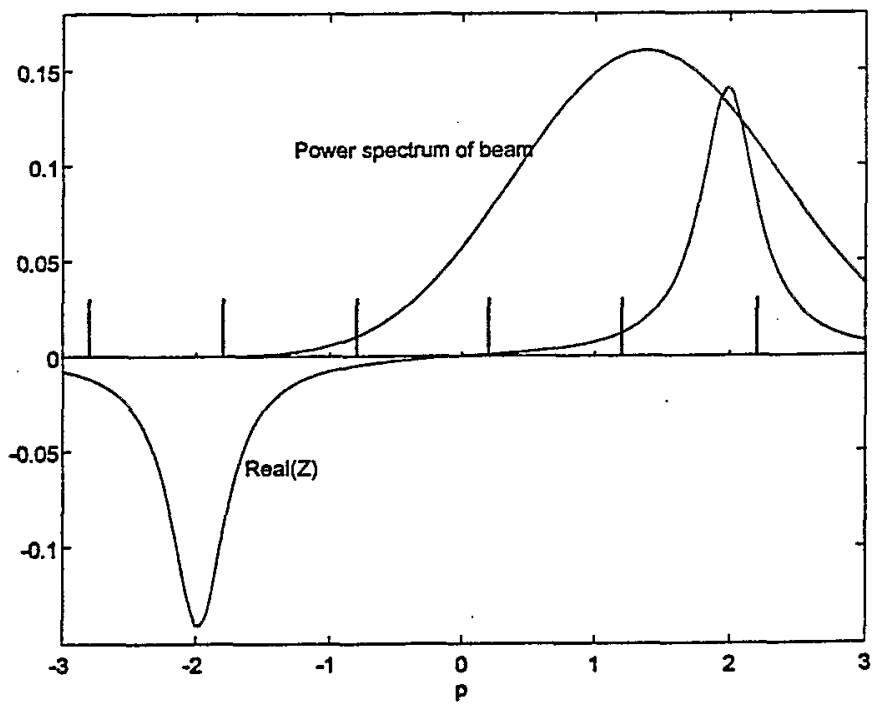

Fig.5. Head-Tail Instability, Narrow Band Resonator 


\section{Multiple Bunch Instabilities}

\subsection{Beam dynamic equation}

To extend the result from the single bunch to the multiple bunch, we need to review the equation (2-24). In the equation, the factor of $Z_{T}(p) \Lambda(p)$ represents the feedback force, and the Bessel function $J_{m}(p r)$ represents the effect of that force applied to the beam. After some manipulations, mainly the integration over the radial $r$, this function gives rise to another spectrum $\Lambda^{(m)}(p)$, as shown in (6-2), which represents the effect of the feedback force.

Consider the multiple bunch case with the harmonic number $h$ and the same revolution frequency. In the time domain, the signal pulses are $h$ times denser, and in the frequency domain, the spectrum pulses are $h$ times sparser. The amplitude of the spectrum, however, is $h$ times larger. This affects the spectrum in $Z_{T}(p) \Lambda(p)$. On the other hand, the mechanism associated with the feedback effect on a given bunch is unchanged. Therefore if the original spectrum $\Lambda_{0}^{(m)}(p)$ is used, the equation (6-2) is modified as,

$$
\omega-\omega_{\beta}-m \omega_{S}=\frac{j e I_{0} h}{2 R m_{0} \gamma \omega_{\beta}} \sum_{p=-\infty}^{\infty} Z_{T}(p) \Lambda_{0}^{(m)}\left(p^{\prime}\right) \Lambda_{0}^{(m)}\left(p^{\prime}\right)
$$

where the added $h$ represents the variation of the amplitude of the spectrum of the signal, and meanwhile, the samplings become at $p h \omega_{0}+\omega_{\beta}+m \omega_{S}$.

\subsection{Coupled bunch instability}

To study the coupled bunch instability, the result of the multiple bunch instability can be modified by replacing the samplings determined by $p h \omega_{0}+\omega_{\beta}+m \omega_{S}$ to $p h \omega_{0}+n \omega_{0}+\omega_{\beta}+m \omega_{S}$, where $n$ is the coupled bunch mode number. Note that the situation considered in (6-3) is for the $n=0$ mode. Consider the AGS with the vertical tune $\nu=8.7$, then the coupled bunch mode $n=7$ is shown in Fig.6. It is observed that this mode is the most unstable one.

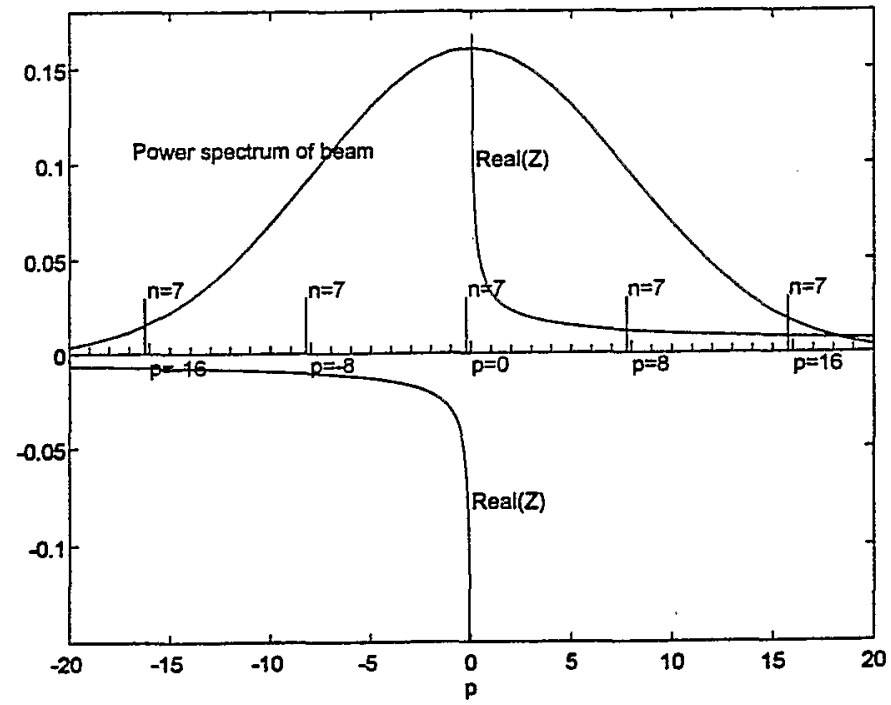

Fig.6. Transverse Coupled Bunch Mode $n=7$

For different chromaticity, the coupled bunch modes are shown in Fig.7. At zero chromaticity, about half modes are stable, and the others are unstable. Below transition, moving 
to negative chromaticity can improve the situation. Larger chromaticity correction, however, may reduce the dynamic aperture and therefore causes beam loss.

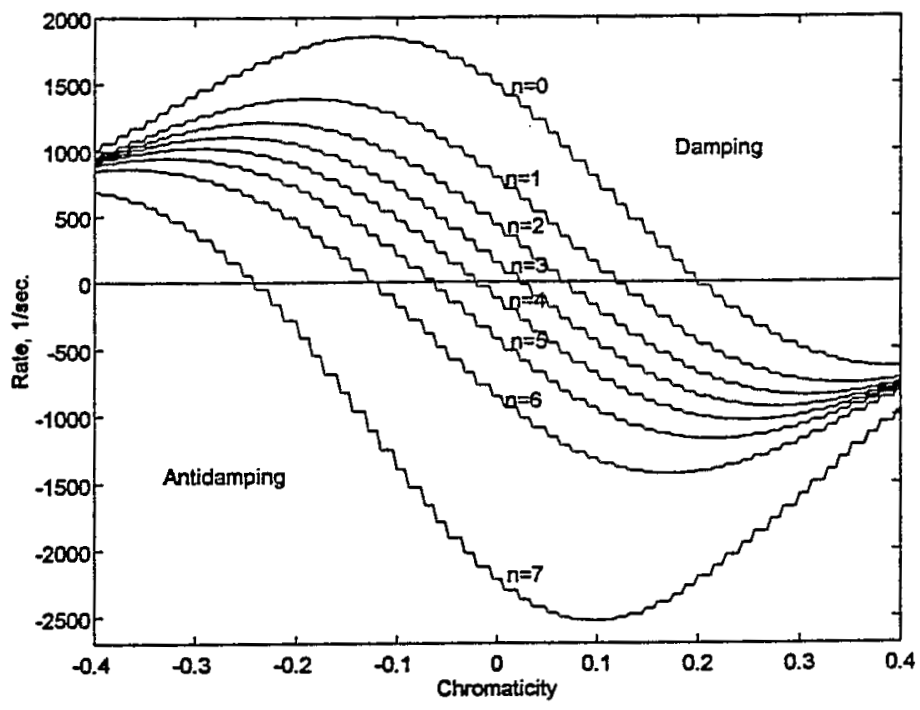

Fig.7. Coupled Bunch Modes, $m=0$

Also for higher order modes, the situation is different. For the same parameters used in Fig.7, the coupled bunch modes of $m=1$ mode are shown in Fig.8.

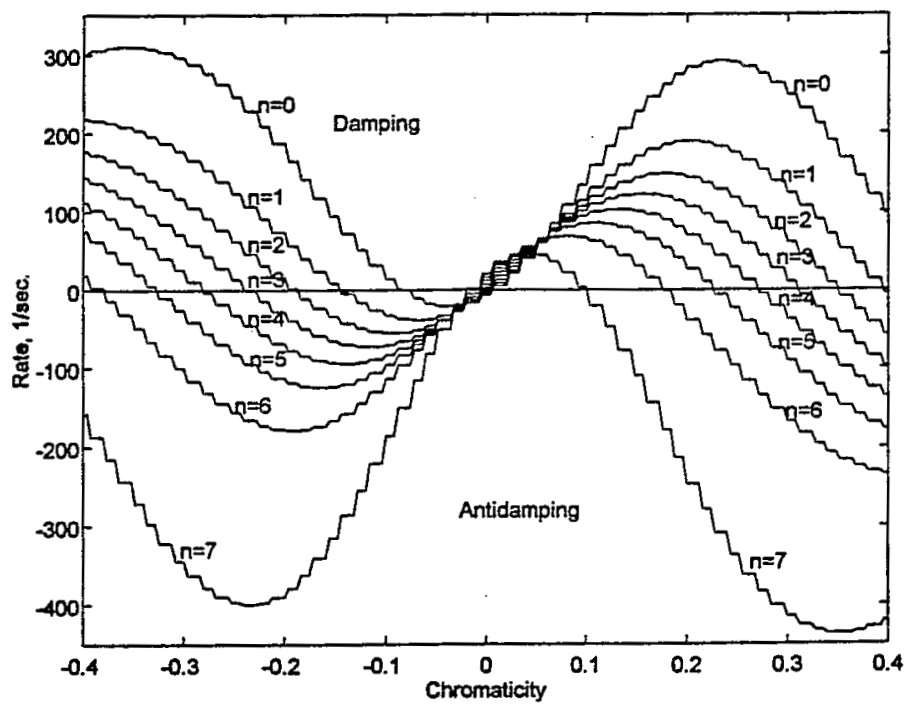

Fig.8. Coupled Bunch Modes, $m=1$

\subsection{Partial coupled bunch instability}

The partial coupled bunch instability refers to an interesting case of the coupled bunch instability of partially filled ring. This is an important operation condition at the AGS where the beam is transferred from the booster in four batches. A coupled bunch mode implies a low frequency mode modulation. At the AGS, if we take $\nu=8.8$, then the $n=7$ coupled bunch mode corresponds merely $69 \mathrm{KHz}$, compared with the betatron oscillation frequency of about $3 \mathrm{MHz}$. This low frequency mode comes from all bunches in a given 
oscillation pattern, and it is highly idealized. In a partially filled ring, the filled part and the gaps in the ring give rise to a low frequency modulation, which is not a single mode but some frequency bands. An example of the AGS first injected batch introduced frequency modulation is shown in Fig.9, where the simple case of the $n=7$ mode is also shown for comparison. For an analysis we need to consider the total effect of this modulation, which includes the folding of the all frequency components of the modulation and their relative phases.

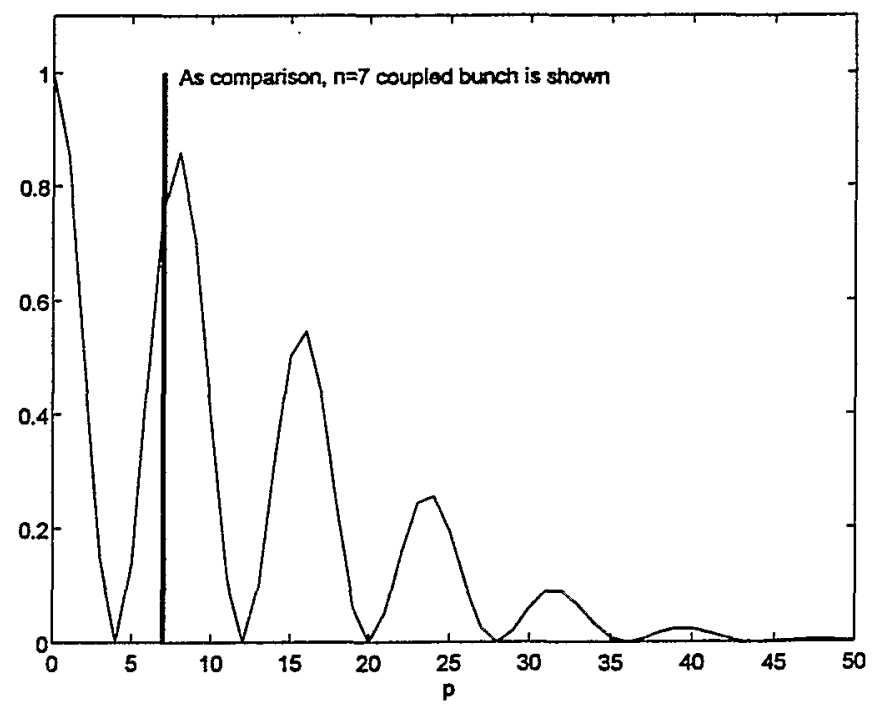

Fig.9. Frequency Modulation of the First Batch at the AGS

\section{Acknowledgment}

The author would like to thank T. Roser for suggesting this subject and for several helpful discussions.

\section{References}

[1] G. Besnier, D. Brandt, and B. Zotter, Particle Accelerators, vol.17, p.51, 1985.

[2] G. Besnier, Nucl. Instru. Methods, vol.164, p.235, 1979.

[3] S.Y. Zhang and W.T. Weng, BNL60557, 1994.

[4] B. Zotter, CERN 85-19, p.253, 1985.

[5] J. Gareyte and F. J. Sacherer, 9th Conf. High Energy Particle Accelerators, 1974.

[6] F.J. Sacherer, 9th Conf. High Energy Particle Accelerators, 1974.

[7] A. Chao, 'Physics of Collective Beam Instabilities in High Energy Accelerators', Wiley, New York, 1994. 